\title{
Investigation of the Local Force Approximation in Numerical Device Simulation by Full-band Monte Carlo Simulation
}

\author{
C. JUNGEMANN*, B. NEINHÜS and B. MEINERZHAGEN \\ Institut für Theoretische Elektrotechnik und Mikroelektronik, Universität Bremen, \\ Kufsteiner Strasse, Postfach 3304 40, 28334 Bremen, Germany
}

\begin{abstract}
The critical assumptions in the drift-diffusion model are the local force approximation and the use of the Einstein relation under nonequilibrium conditions. The validity of these two approximations is investigated by full-band Monte Carlo simulation for a SiGe-HBT. It is found that neither the local force approximation nor the Einstein relation holds. Even Einstein relations generalized with the local temperature fail under quasiballistic transport conditions, indicating that the energy transport and hydrodynamic approach are also problematic.
\end{abstract}

Keywords: Full-band Monte Carlo; Drift-diffusion; Local force approximation; Transport parameters; Einstein relation; SiGe, HBT

\section{INTRODUCTION}

The classical drift-diffusion (DD) model is the workhorse of today's TCAD. It is based on transport coefficients parameterized with the local force (i.e., gradient of the quasifermi potential), where the transport parameters are determined under homogeneous bulk conditions [1]. The DD model is based on the assumptions that the local distribution function in the device is completely determined by the local force and that the Einstein relation holds. The validity of these two assumptions is investigated in this work by full-band Monte Carlo (FB-MC) device simulation. In contrast to earlier works [2] the investigation is directly based on the exact transport coefficients [3] evaluated under local and nonlocal conditions by FB-MC simulations instead of merely comparing velocity and density profiles of the DD and FBMC models. First, a brief discussion of the theoretical background of the DD model is given and then the DD model is investigated for a SiGe-HBT.

*Corresponding author. Tel.: +49 421218 4681, Fax: +49 421218 4434, e-mail: junge@item.uni-bremen.de 


\section{THEORY}

In the framework of semiclassical transport theory the electron gas in a semiconductor under stationary conditions is described by the following Boltzmann transport equation (BTE) [4]

$$
\begin{aligned}
& \left\{\vec{F}_{n}^{\mathrm{T}}(\vec{r}, \vec{k}) \frac{1}{\hbar} \nabla_{\vec{k}}+\vec{v}_{n}^{\mathrm{T}}(\vec{r}, \vec{k}) \nabla_{\vec{r}}\right\} f_{n}(\vec{r}, \vec{k}) \\
& =\frac{\Omega}{(2 \pi)^{3}} \sum_{n^{\prime}} \int_{\mathrm{BZ}} S_{n, n^{\prime}}\left(\vec{k} \mid \vec{k}^{\prime}\right)(\vec{r}) f_{n^{\prime}}\left(\vec{r}, \vec{k}^{\prime}\right) \\
& \quad-S_{n^{\prime}, n}\left(\vec{k}^{\prime} \mid \vec{k}\right)(\vec{r}) f_{n}(\vec{r}, \vec{k}) d^{3} k^{\prime},
\end{aligned}
$$

where the force $\vec{F}$ is given by $-q \vec{E}(\vec{r})-\nabla_{\vec{r}}\left(E_{\mathrm{c}}(\vec{r})+\right.$ $\left.\varepsilon_{n}(\vec{r}, \vec{k})\right) . q$ is the positive electron charge, $\vec{E}$ the electric field, $E_{\mathrm{c}}$ the minimum of the first conduction band, and $\varepsilon$ the position-dependent conduction band energy relative to the minimum. $\vec{v}$ is the particle velocity $\left(1 / \hbar \nabla_{\vec{k}} \varepsilon\right), f$ the distribution function, $\Omega$ the system volume, and $S$ the transition rate. $\mathrm{BZ}$ denotes the Brillouin zone.

The DD model is based on balance equations for two moments of the BTE $[1,4,5]$. The balance equations are obtained by multiplying the BTE from the left with the respective microscopic quantity and calculating the position-dependent expectations. The continuity equation is obtained with the microscopic quantity 1

$$
\nabla_{\vec{r}}^{\mathrm{T}} \vec{j}=0
$$

neglecting generation and recombination phenomena. The particle current density $\vec{j}$ is given by $n\langle\vec{v}\rangle$, where $n$ is the electron density and the expectation $\langle\vec{v}\rangle$ is obtained by integrating over the $\mathrm{BZ}$ and summing over all conduction bands. The expectations are normalized $\langle 1\rangle=1$. With the microscopic relaxation time $\tau$ defined by [6]

$$
\begin{aligned}
\vec{v}_{n}(\vec{r}, \vec{k})= & \frac{\Omega}{(2 \pi)^{3}} \sum_{n^{\prime}} \int_{\mathrm{BZ}} S_{n^{\prime}, n}\left(\vec{k}^{\prime} \mid \vec{k}\right)(\vec{r}) \\
& {\left[\tau_{n}(\vec{r}, \vec{k}) \vec{v}_{n}(\vec{r}, \vec{k})-\tau_{n^{\prime}}\left(\vec{r}, \vec{k}^{\prime}\right) \vec{v}_{n^{\prime}}\left(\vec{r}, \vec{k}^{\prime}\right)\right] d^{3} k^{\prime}, }
\end{aligned}
$$

the stationary balance equation for the quantity $\tau \vec{v}$ reads

$$
\begin{aligned}
\vec{j}= & -n \hat{\mu}\left(\vec{E}+\frac{1}{q} \nabla_{\vec{r}} E_{\mathrm{c}}\right) \\
& -\left(\nabla_{\vec{r}}^{\mathrm{T}}[n \hat{D}]\right)^{\mathrm{T}}+\left\langle\left(\nabla_{\vec{r}}\left[\tau \vec{v}^{\mathrm{T}}\right]\right)^{\mathrm{T}} \vec{v}\right\rangle \\
& -n\left\langle\frac{1}{\hbar} \nabla_{\vec{k}}\left[\tau \vec{v}^{\mathrm{T}}\right] \nabla_{\vec{r} \varepsilon}\right\rangle,
\end{aligned}
$$

where the matrix-valued mobility $\hat{\mu}$ and diffusion constant $\hat{D}$ are given by [3]

$$
\hat{\mu}=\left\langle\frac{q}{\hbar} \nabla_{\vec{k}}\left[\tau \vec{v}^{\mathrm{T}}\right]\right\rangle
$$

and

$$
\hat{D}=\left\langle\tau \vec{v}^{\mathrm{T}}\right\rangle
$$

Both quantities are symmetric for the given scattering processes. It is worth noting that Eqs. (2) and (4) are obtained without any approximations.

Close to equilibrium and for a band energy which can be factored into a position- and wavevector-dependent part $\varepsilon_{n}(\vec{r}, \vec{k}) \approx g(\vec{r}) h_{n}(\vec{k})$ Eq. (4) reduces to [4]

$$
\vec{j}=n \hat{\mu} \nabla_{\vec{r}} \Phi
$$

where the quasifermi potential is defined by

$$
n=n_{i} \exp \left(q \frac{\Psi-\Phi}{k_{B} T_{0}}\right)
$$

The intrinsic density $n_{\mathrm{i}}$ is evaluated with the position-dependent band structure. $\Psi$ is the electrostatic potential, $k_{\mathrm{B}}$ the Boltzmann constant, and $T_{0}$ the lattice temperature.

In the case of the DD model Eq. (7) is also used under hot-electron conditions instead of Eq. (4). Since the distribution function can not be evaluated by the DD model, the mobility (5) is assumed to be a function of the absolute value of the local gradient of the quasifermi potential

$$
\vec{j}=n \hat{\mu}\left(\left|\nabla_{\vec{r}} \Phi\right|\right) \nabla_{\vec{r}} \Phi
$$


and is evaluated under homogeneous bulk conditions. Thus, it is assumed that the distribution function in a device is fully characterized by the local force. In addition, Eq. (7) is based on the assumption that the Einstein relation valid in equilibrium

$$
\hat{D}=\hat{\mu} \frac{k_{\mathrm{B}} T_{0}}{q}
$$

also holds in strong nonequilibrium with the lattice temperature.

\section{RESULTS}

The validity of the two approximations given above is investigated by comparison of transport parameters calculated with the device distribution function and with the one for a bulk system with the corresponding force. To this end 1D FB-MC device simulations of a SiGe-HBT and corresponding bulk simulations are performed. The electron density and velocity are shown in Figure 1 and a strong velocity overshoot is found in the vicinity of the base/collector junction ( $x=$ $0.32 \mu \mathrm{m})$. In Figure 2 the local temperature and force are shown, where the local force is defined as the ratio of the drift velocity and mobility

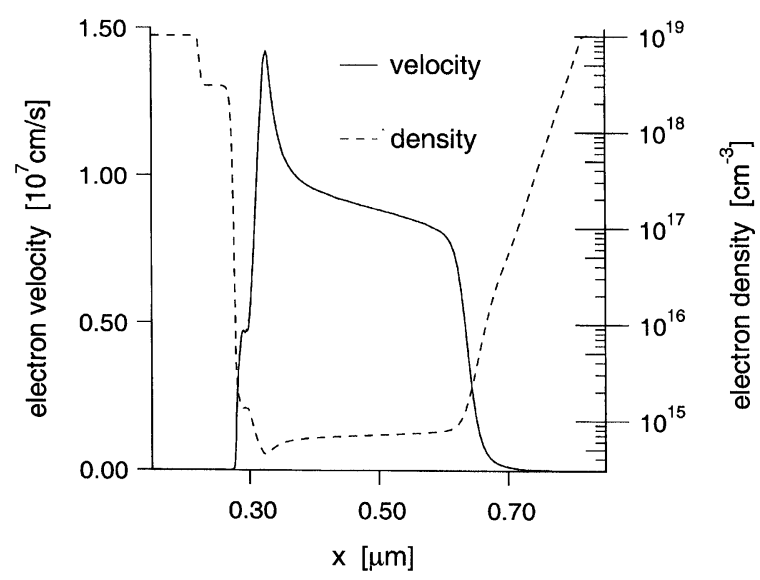

FIGURE 1 Electron density and velocity for a SiGe-HBT with $V_{\mathrm{CE}}=2.70 \mathrm{~V}$ and $V_{\mathrm{BE}}=0.70 \mathrm{~V}$.

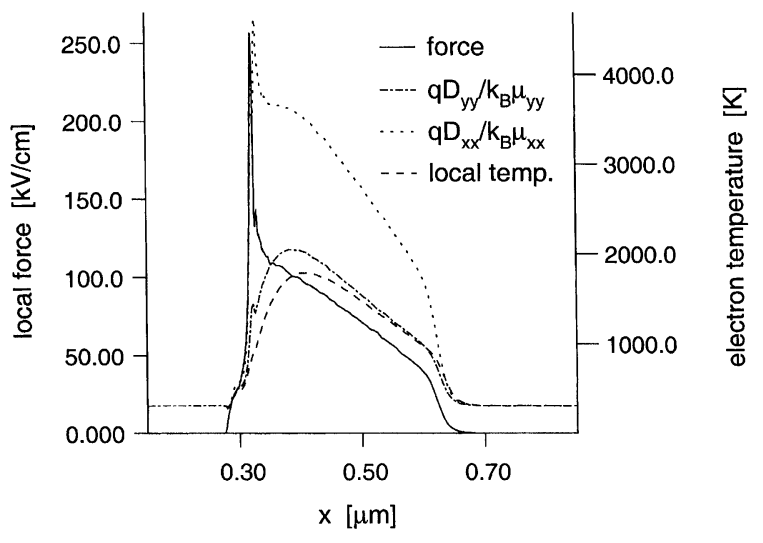

FIGURE 2 Local force, local temperature, and temperatures derived by generalized Einstein relation.

$\left(\partial \Phi / \partial x=v_{x} / \mu_{x x}\right)$. In Figure 3 the ratio of the longitudinal mobility calculated under homogeneous conditions for the local force within the device and evaluated based on the device distribution function in the device with Eq. (5) is shown. As expected, the nonlocal mobility shows a strong overshoot corresponding to the velocity overshoot.

In order to incorporate nonlocal transport effects into the DD model, the local mobility/force

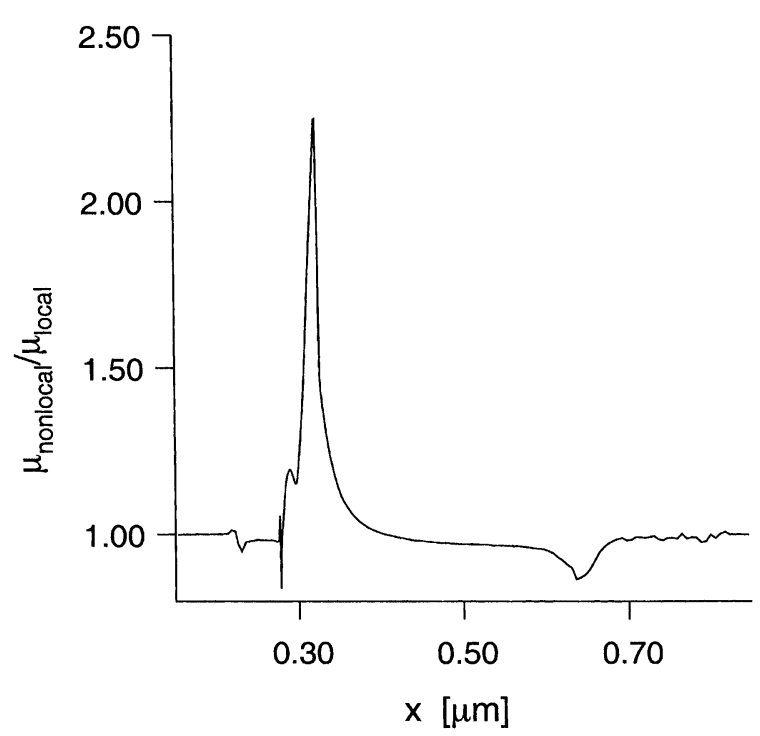

FIGURE 3 Ratio of the nonlocal mobility and the mobility calculated for the local force. 
relation of the DD model is multiplied with the ratio of Figure 3 for each cell of the real space grid. In Figure 4 the resultant velocity profile together with the original DD and FB-MC velocity is shown. The modified DD (MDD) model reproduces the FB-MC result quite well including the velocity overshoot. This is reflected in the electron density (Fig. 5). Unfortunately, this does not mean that the overall simulation results are improved. The collector current, for example, is worse: $I_{\mathrm{MC}}=$ $100 \mathrm{~mA}, \quad I_{\mathrm{DD}}=125 \mathrm{~mA}, \quad I_{\mathrm{MDD}}=144 \mathrm{~mA}$. Thus, simple incorporation of nonlocal transport effects does not necessarily improve the DD model. One reason for this is that the DD model is based on the Einstein relation which yields the wrong diffusion constant. In Figure 6 the longitudinal $\left(\mu_{x x}\right)$ and transverse $\left(\mu_{y y}\right)$ mobility are shown together with the respective diffusion constants divided by $k_{\mathrm{B}} T_{0} / \mathrm{q}=0.0263 \mathrm{~V}\left(T_{0}=305 \mathrm{~K}\right)$. If the Einstein relation were to hold, the mobilities and the corresponding diffusion constants would agree well. This is only the case in regions with a high electron concentration, where the electron gas is close to equilibrium. Furthermore, the difference in mobility and diffusion constant is not

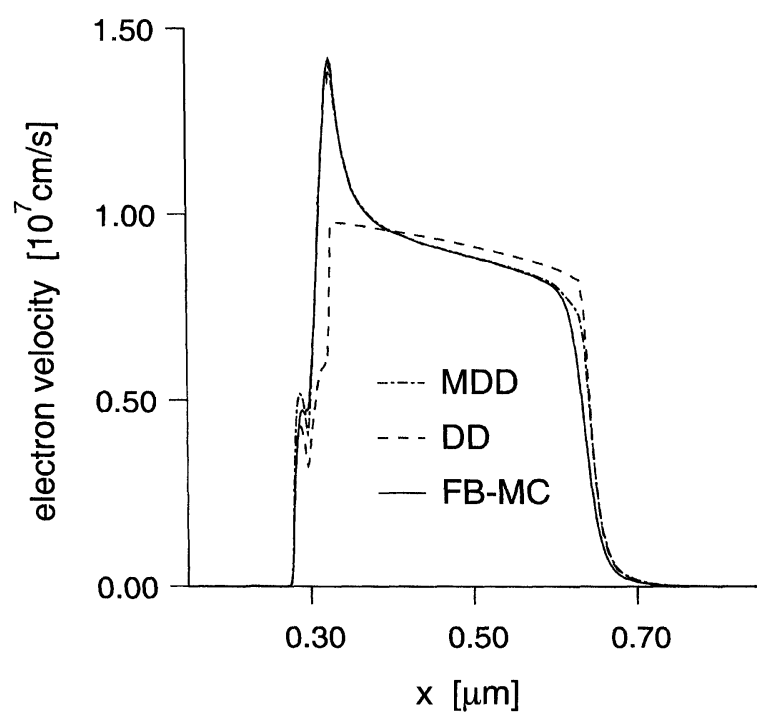

FIGURE 4 Electron velocity calculated by FB-MC, DD, and MDD.

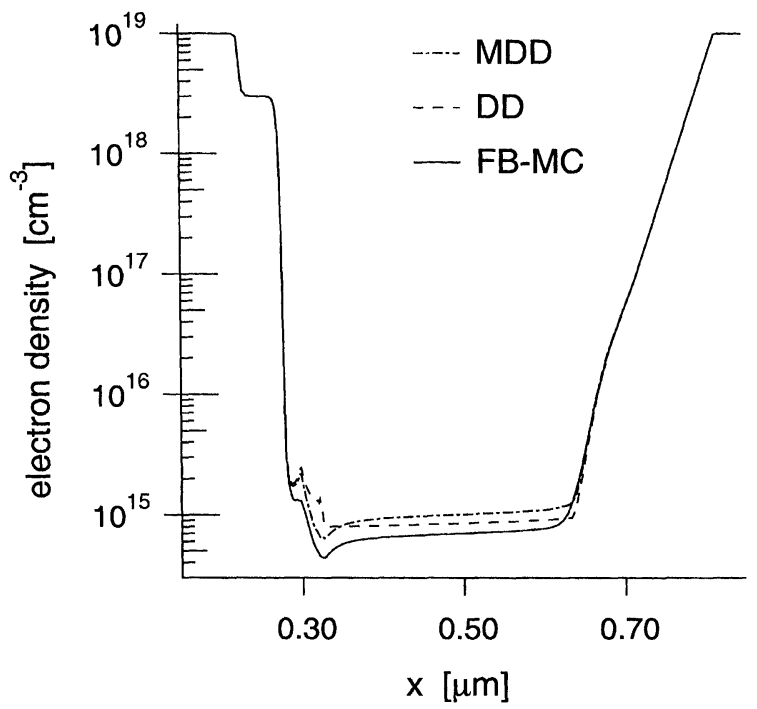

FIGURE 5 Electron density calculated by FB-MC, DD, and MDD.

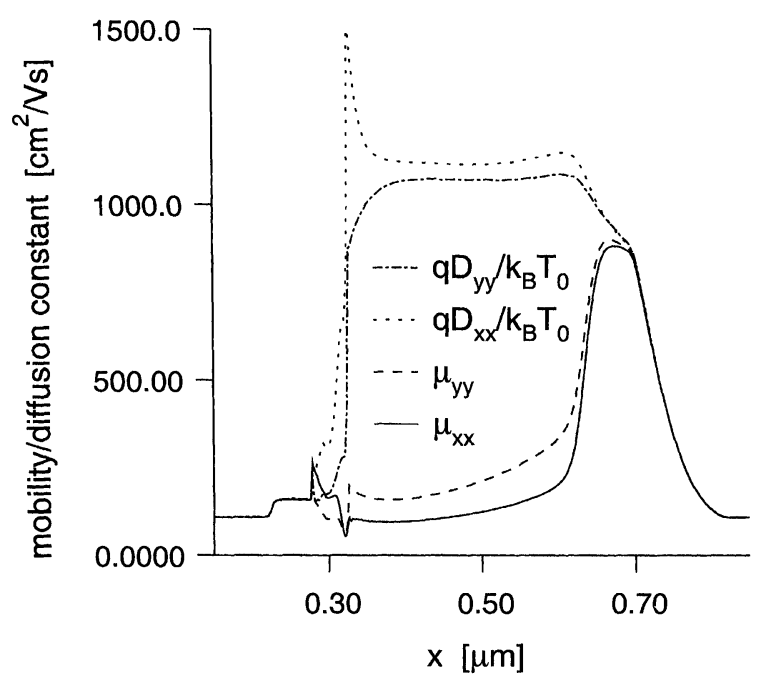

FIGURE 6 Longitudinal and transverse mobility and diffusion constant divided by $k_{\mathrm{B}} T_{0} / q$.

simply explained by the local temperatures as shown in Figure 2, where the local temperature, $q D_{\mathrm{xx}} / \mathrm{k}_{\mathrm{B}} \mu_{x x}$, and $q D_{\mathrm{yy}} / \mathrm{k}_{\mathrm{B}} \mu_{y y}$ are shown. Whilst the transverse transport is reasonably described by a generalized Einstein relation using the local temperature instead of the lattice temperature, the more important longitudinal transport is not. 
This also means that the energy transport or hydrodynamic approaches will not capture all details of the nonlocal transport by using a generalized Einstein relation based on the local temperature.

\section{CONCLUSIONS}

The quality of the DD approximation is investigated for the first time with transport parameters evaluated under nonlocal conditions by FB-MC device simulation for an SiGe-HBT. But not only the failure of the local force approximations is demonstrated but also the failure of generalized Einstein relations based on the local temperature. This calls into question the validity of the energy transport or hydrodynamic approach. Moreover, strong anisotropy of the transport coefficients is found.

\section{Acknowledgments}

This work was supported in part by the Bundesministerium für Bildung, Wissenschaft, Forschung und Technologie under Contract No. 01M2416A.

\section{References}

[1] Selberherr, S., Analysis and Simulation of Semiconductor Devices, Springer, Wien, 1984.

[2] Tomizawa, M., Yokoyama, K. and Yoshii, A. (1998). "Nonstationary carrier dynamics in quarter-micron $\mathrm{Si}$ MOSFETs", IEEE Trans. Computer-Aided Des., CAD-7, 254-258.

[3] Price, P. J. (1979). "Monte Carlo calculation of electron transport in solids", Semiconductors and Semimetals, 14, 249-309.

[4] Marshak, A. H. and van Vliet, K. M. (1978). "Electrical current in solids with position-dependent band structure", Solid-State Electron., 21, 417-427.

[5] Stratton, R. (1962). "Diffusion of hot and cold electrons in semiconductor barriers", Phys. Rev., 126, 2002-2013.

[6] Brauer, W. and Streitwolf, H. W. (1977). Theoretische Grundlagen der Halbleiterphysik, Vieweg, Braunschweig, 2nd edition. 

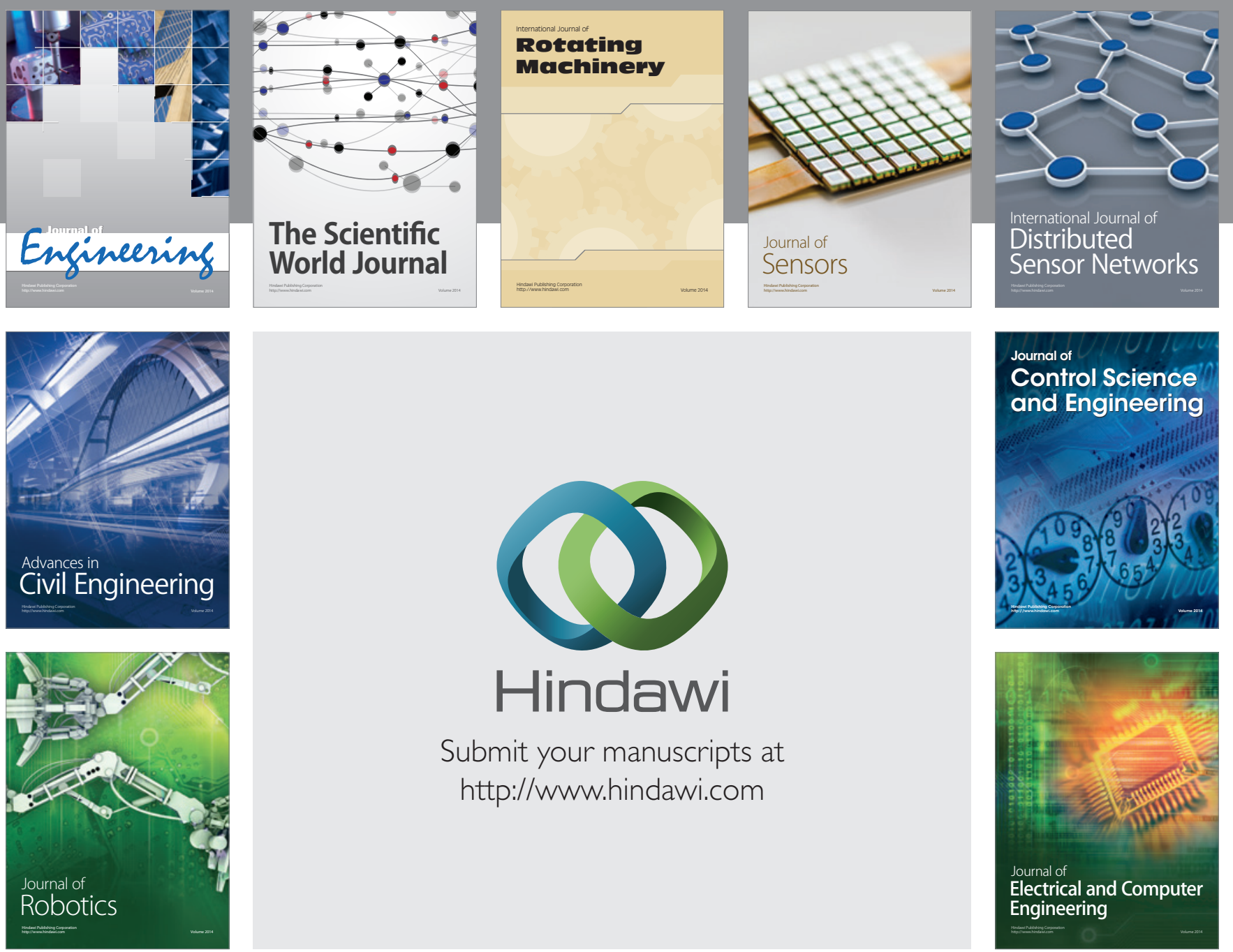

Submit your manuscripts at

http://www.hindawi.com
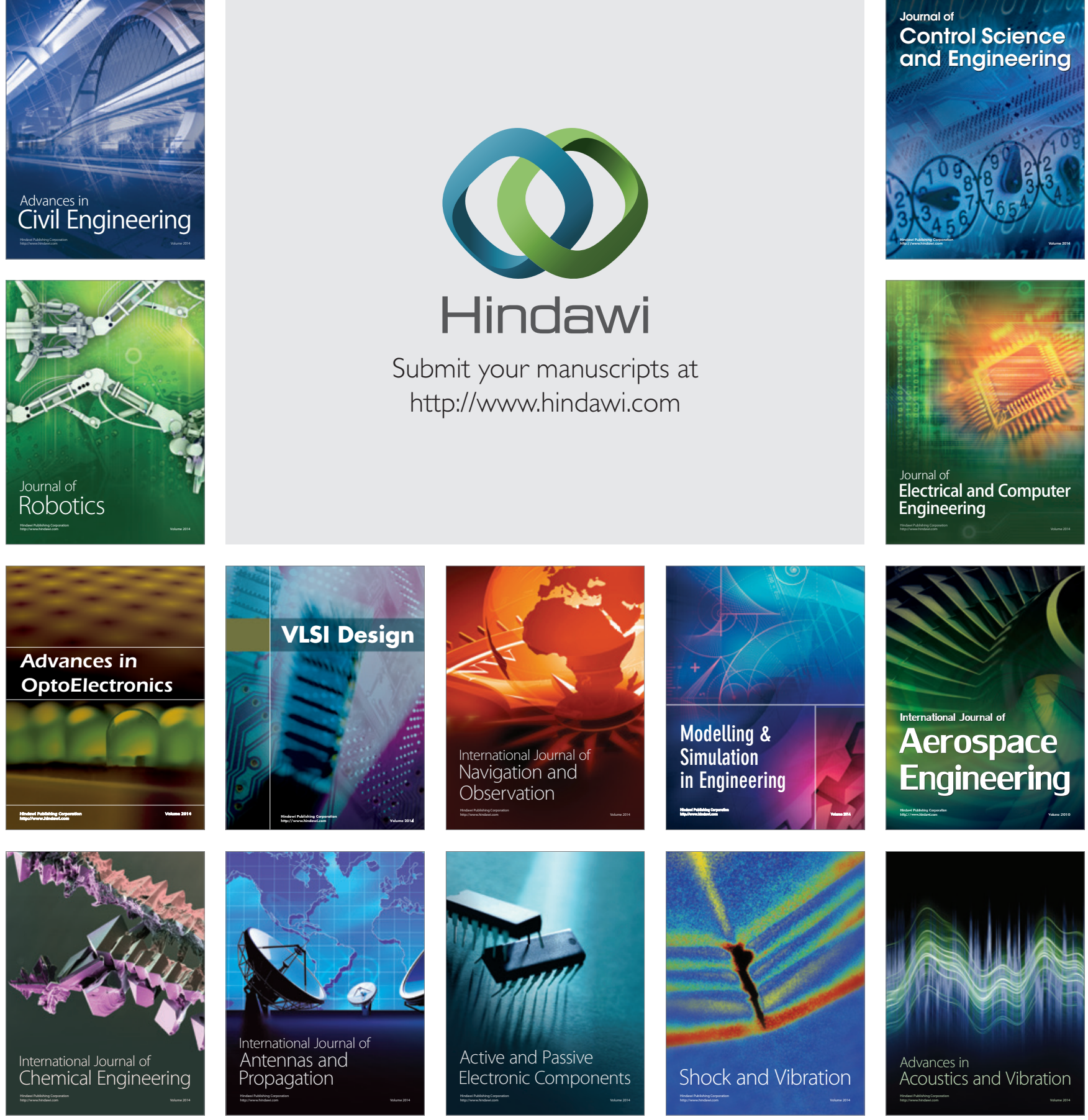